\title{
EXTRA INTERPHALANGEAL TRANSVERSE CREASES OF THE LITTLE FINGER
}

\author{
Yosuke Komatz, Kazuyuki DaIJo, and Osamu Yoshida \\ The Department of Urology, Faculty of Medicine, Kyoto \\ University, Sakyo-ku, Kyoto 606, Japan
}

\begin{abstract}
Summary A series of 283 male and 268 female individuals were examined for digital flexion creases on the little fingers. Two males and two females were found to have an extra interphalangeal transverse crease situated betweeen the metacarpophalangeal and proximal interphalangeal creases. The extra crease of the little finger was unilateral or bilateral. None of the little fingers with extra crease had radiographic anomalies of the bones or joints, or evidence of dyskinesia.
\end{abstract}

Three digital flexion creases are normally observed to exist on the little finger, i.e. metacarpophalangeal crease and proximal and distal interphalangeal creases. A ring crease, which is usually present as a metacarpophalangeal crease on the middle finger and ring finger, is only rarely found on the little finger (Okajima, 1966).

A single interphalangeal crease on the little finger represents, as generally recognized, a characteristic dermatoglyphic trait in Down's syndrome, 18-trisomy, de Lange syndrome and oral-facial-digital syndrome (Alter, 1966; Preus and Fraser, 1972). The defect of one of the digital creases of little finger in these conditions, therefore, has been attracting attention of many investigators.

There is a dearth of detailed information of cases with an extra transverse digital crease on the little finger, in addition to the usual three digital creases. We examined a series of 551 individuals for the extra transverse flexion crease of the little fingers (1102 fingers). Four subjects were found to have this trait located between the proximal interphalangeal crease and metacarpophalangeal crease. The present communication deals with the observation of extra interphalangeal transverse creases on the little fingers in these four cases.

\section{MATERIALS AND METHODS}

A total of 1102 little fingers of 283 males and 268 females were studied. The subjects consist of 199 male and 85 female urological patients, 69 males and 42 females from 15 male and 141 female spouses of the patients and their relatives, 

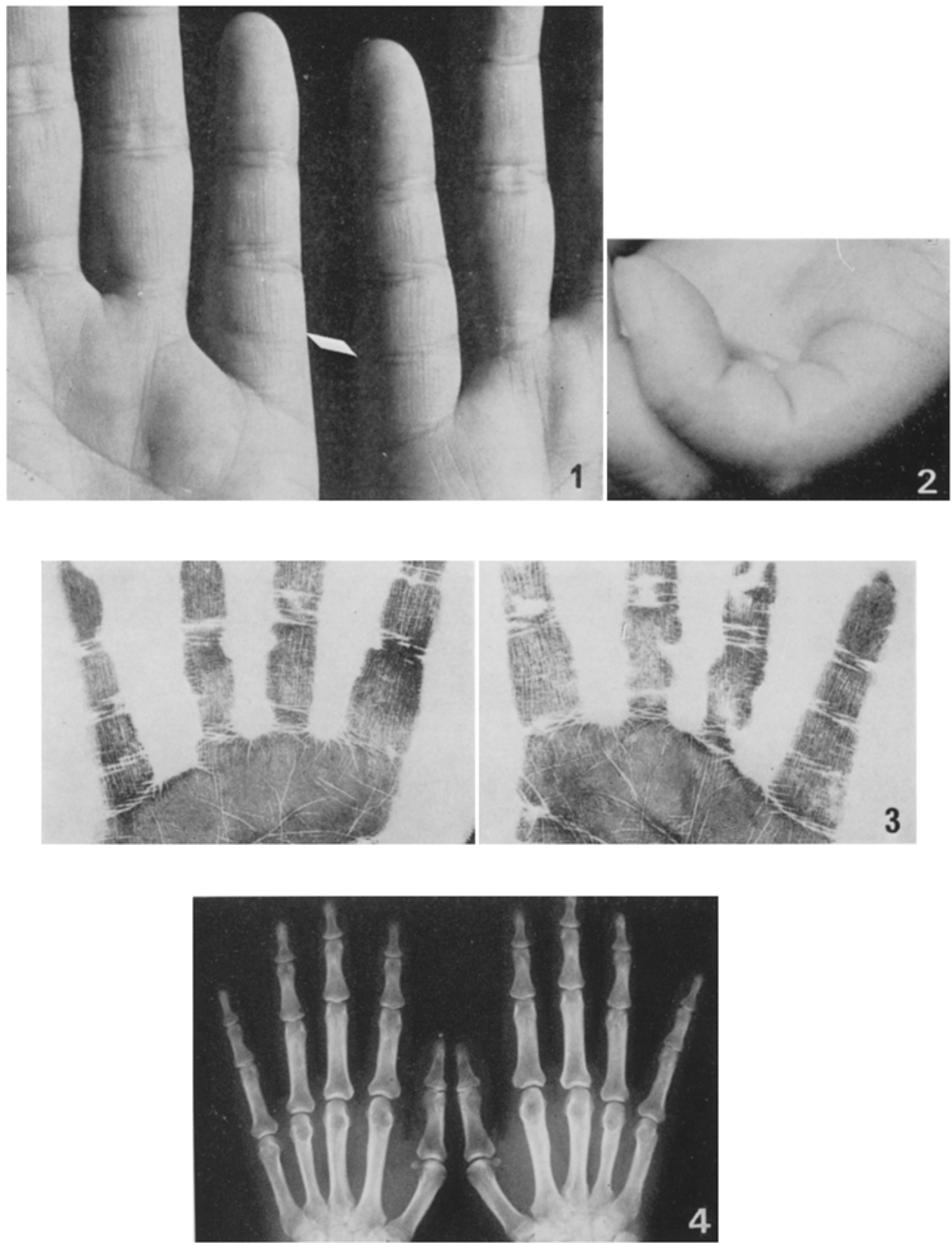

Fig. 1. Case 1. Bilateral case. Extra transverse creases located between the proximal interphalangeal crease and the metacarpophalangeal crease of the little finger.

Fig. 2. Case 1. Four flexion lines formed on flexing the little finger.

Fig. 3. Case 1. Fingerprints. Extra transverse creases bear morphological resemblance to the interphalangeal creases.

Fig. 4. Case 1. Radiologically the little fingers had no bone anomalies. 
and physicians and nurses of the Kyoto University Hospital, medical students and students of the School of Nursing of this University. The age ranges for males from 10 to 87 years and for females from 7 to 79 years. Those found to have extra creases on the little fingers were radiologically examined for abnormalities in phalanx or interphalangeal joint.

\section{RESULTS}

Of the 1102 little fingers of 551 individuals studied, six little fingers were noted to show an extra transverse crease, bilateral in 2 females and unilateral in 2 males.

\section{a) Bilateral cases}

Case 1. The subject was a 33-year-old housewife, a mother of two children, who visited this clinic for treatment of a left simple renal cyst. Examination revealed four digital flexion creases (Fig. 1) on each of her little fingers, forming four flexion lines on flexion of each little finger (Fig. 2). The distal two of these four flexion creases coincided respectively, with the distal and proximal interphalangeal joints. The most proximal crease was considered to be a metacarpophalangeal crease. An extra transverse crease was observed to exist between the proximal interphalangeal crease and the metacarpophalangeal crease (Fig. 3). It was parallel to the interphalangeal crease and comprised of one transverse crease and two accesory creases, bearing morphological resemblance not so much to the metacarpophalangeal creases as to the interphalangeal creases. Roentgenograms of both hands did not reveal any significant abnormal findings for the bones of the little fingers (Fig. 4). These extra creases showed crease indices of 56.6 (left) and 59.3 (right) when calculated by the formula of Okajima (1966) for the ring crease index of the middle finger.

None of the patients siblings, parents or two children was found to have extra creases of the little fingers.

Case 2. The subject was a healthy housewife, spouse of the urological patient's son aged 35 years, a mother of two children. Single transverse creases were noted to be present distal to the metacarpophalangeal crease of both little fingers (Fig. 5). Four flexion lines formed on each little fingers with flexion (Fig. 6). Unlike those in Case 1, her extra transverse creases bore striking morphological resemblance to ring creases of the middle and ring fingers and crossed over the phalanx at essentially the same gradient as the metacarpophalangeal creases of the little fingers (Fig. 7). Their crease indices were calculated to be 65.0 (left) and 63.2 (right), respectively.

There was no radiographic evidence of osseous abnormalities in the little fingers, nor were any her siblings, parents or two children found to have such extra creases on the little fingers.

\section{b) Unilateral cases}

Case 3. The subject was a 31-years-old male, infertile for two years since 

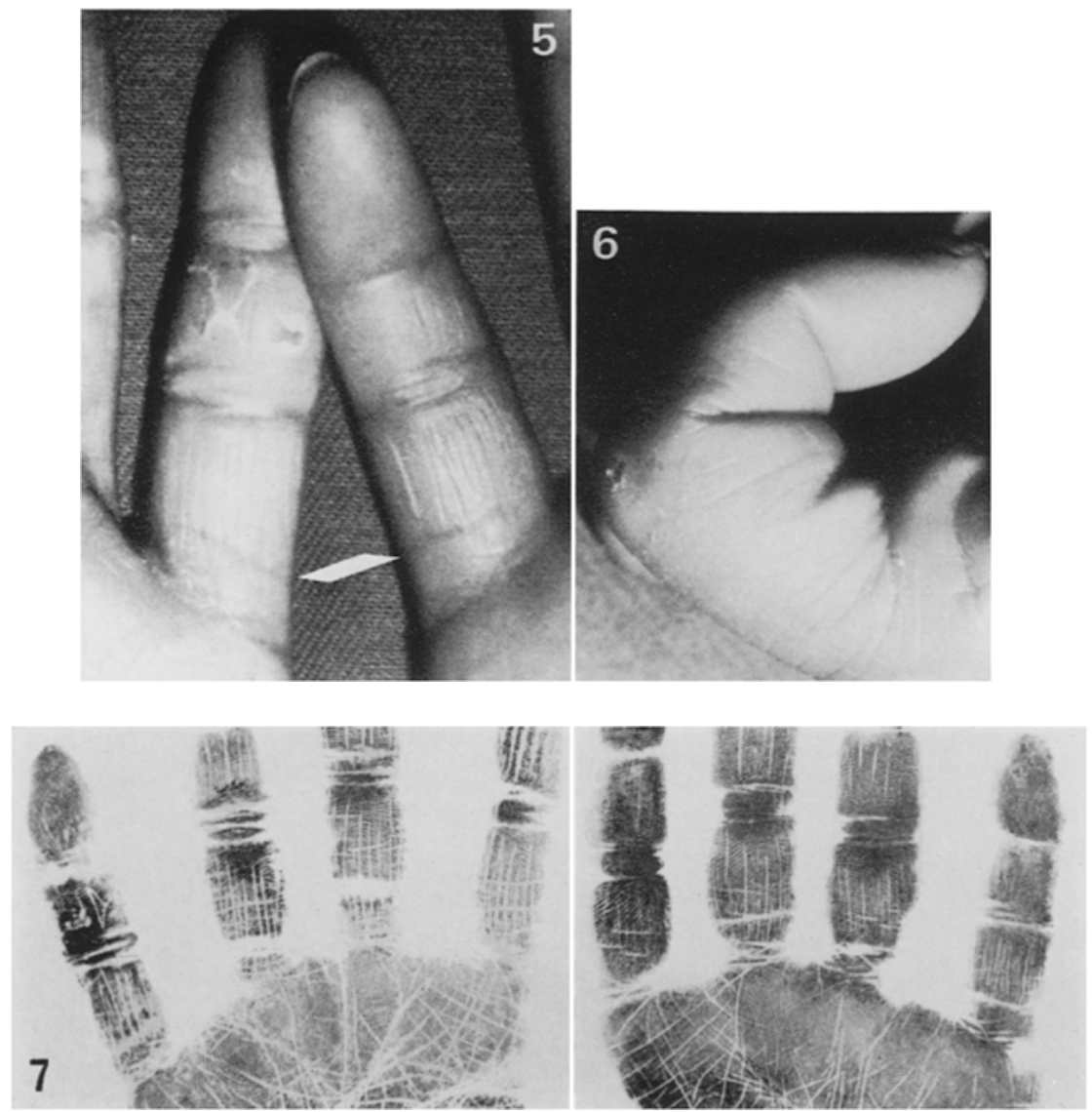

Fig. 5. Case 2. Bilateral case. Extra creases crossing over the little finger at the same gradient as the metacarpophalangeal creases.

Fig. 6. Case 2. Four flexion lines.

Fig. 7. Case 2. Fingerprints show that extra creases resemble the ring creases of the middle and ring fingers.

marriage. Seminal analysis disclosed azoospermia, which, together with the normal histologic findings on testicular biopsy, suggested a probable diagnosis of obstructive azoospermia. Dermatoglyphic examination of the hands revealed the presence of an extra transverse crease between the metacarpophalangeal and proximal interphalangeal creases of the right little finger (Figs. 8 and 9). No such extra crease was found on the left little finger. The extra crease had morphological resemblance to the metacarpophalangeal crease rather than otherwise (Fig. 10) and was found to show a crease index of 56.5. The little fingers had no bone anomalies on radiologic examination. No information has been obtained as to creases or structures of fingers of his parents or siblings.

Case 4. The subject was a man of 40 years of age, presumed to be infertile for 

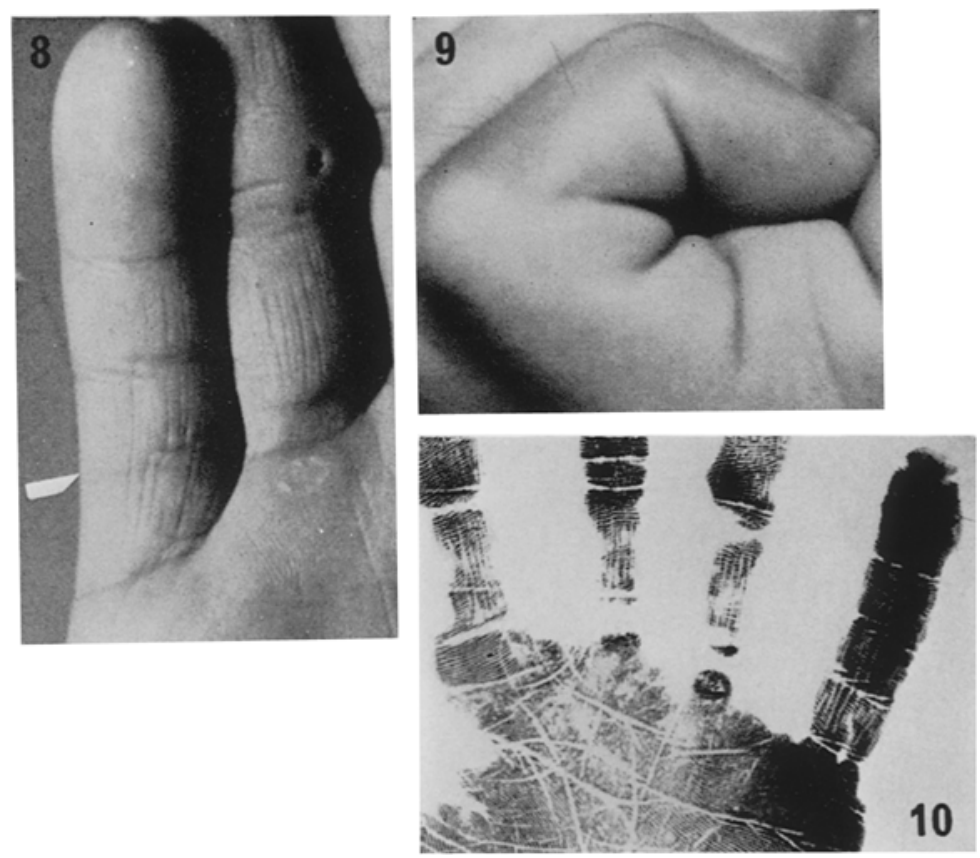

Fig. 8. Case 3. Unilateral case. Extra transverse crease of the right little finger.

Fig. 9. Case 3. Four flexion lines.

Fig. 10, Case 3. Fingerprints.

15 years since marriage. Seminal analysis showed a normal fertile semen. An extra transverse crease was found between the metacarpophalangeal and proximal interphalangeal creases of the right little finger (Figs. 11 and 12), bearing morphological resemblance to the metacarpophalangeal crease (Fig. 13) and showing a crease index of 50.0 Radiographic examination showed no anomalies in the structure of little fingers. No information has been obtained concerning the little fingers of his parents or siblings.

\section{DISCUSSION}

A case of extra transverse digital creases was briefly described by Furuya $e t$ al. (1967). The 52-year-old healthy woman had an extra crease on the left little finger which, according to the investigators, was regarded as a ring crease. Her example is similar to Cases 3 and 4 reported herein in that the extra crease was unilateral and located transversely about mid-way between the metacarpophalangeal and proximal interphalangeal creases.

We report the incidence of extra transverse creases on the little finger of males is two out of 566 fingers or $0.35 \%$. Considering the extra creases occurring on the right little fingers alone, the frequency of male individuals with this aberration was 

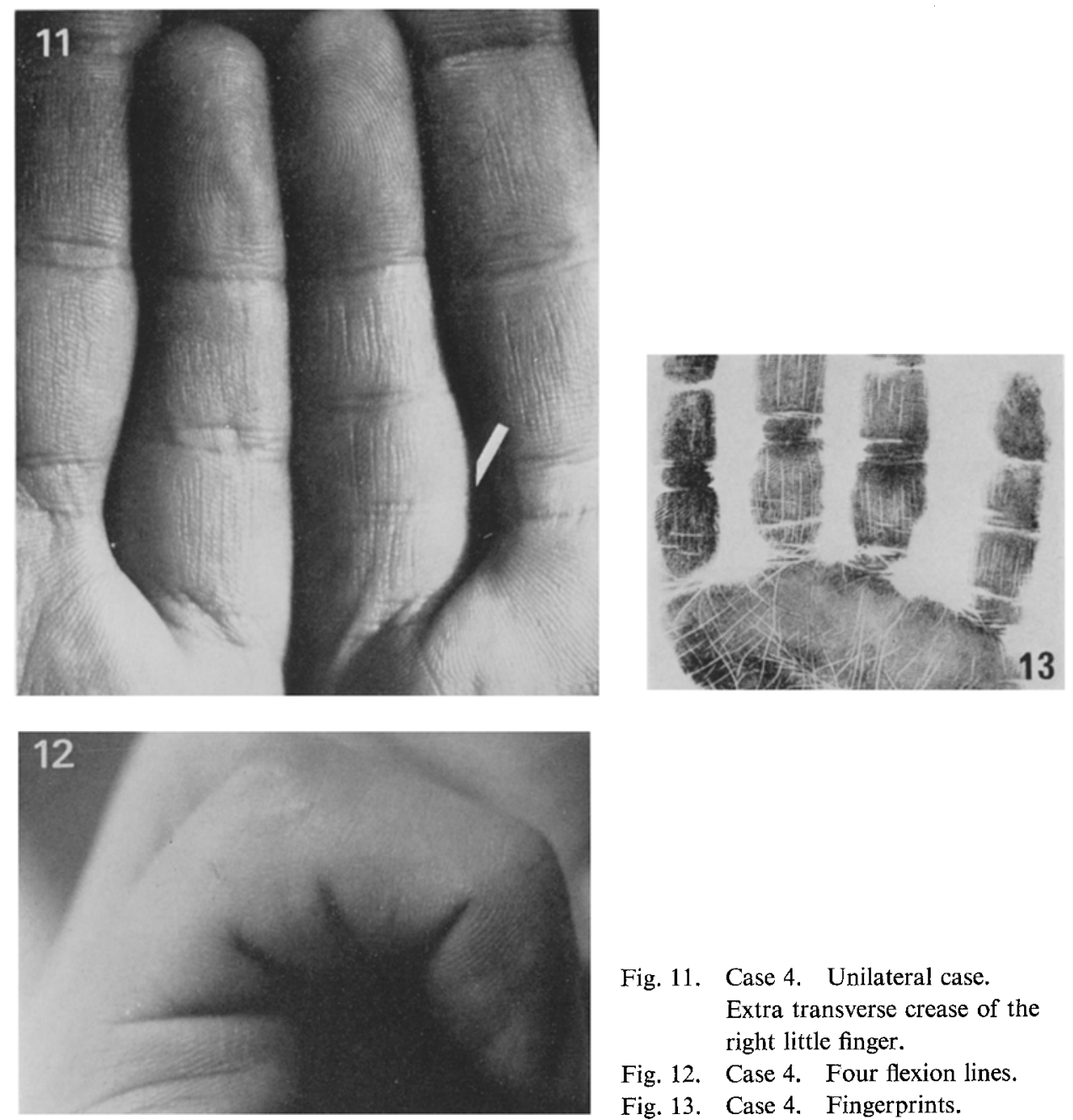

Fig. 11. Case 4. Unilateral case.

Extra transverse crease of the right little finger.

Fig. 12. Case 4. Four flexion lines.

Fig. 13. Case 4. Fingerprints.

two out of 283 subjects or $0.71 \%$, of 268 females, two women had extra creases on both little fingers, with the frequency of occurrence in these individuals of $0.75 \%$. The extra crease of the little finger occurs unilaterally or bilaterally. Unilateral crease may occur in both sexes as it was observed in males in this study while Furuya et al. (1967) reported a female case. Bilateral creases were encountered in females, but not in males.

Extra transverse creases of the little finger are situated in all instances between the metacarpophalangeal crease and proximal interphalangeal crease. They can be classified into two types according to their distance from the metacarpophalangeal crease, viz. one located at a position corresponding to the ring crease described by Okajima (1966) for metacarpophalangeal creases on the middle and ring fingers, 
the other situated considerably distally to the position of ring crease. The crease seen in Case 2 belongs to the former type whilst those in Case 1, 3 and 4 come under the latter category. Okajima (1966) calculated indices for ring creases of middle fingers on the basis of the data he obtained, which ranged between 62.0 and 92.0. When calculated by the same method, the crease index for the extra crease of the little finger in Case 2 was found to fall within this range, whereas the indices in the other three cases were far below the lower limit of the ring index.

The term "ring crease" stems from the fact that this crease is situated at the position on the finger where a finger ring is worn. If a finger ring is worn on the little finger, it may be set at the position of the extra crease found in Case 2 but not at the position of extra creases seen in the remaining three cases. To terminological confusion, it appears reasonable to designate the extra crease in Case 2 as a ring crease of the little finger and the creases in the other three cases and in Furuya's et al. (1967) case as extra interphalangeal transverse creases, respectively.

It has been reported that an extra crease distal to the distal interphalangeal crease of the middle finger is frequently seen in patients with sickle cell disease (DeJong and Plato, 1967). Of the four subjects found to have an extra transverse crease on the little finger in the present study, two were patients with urological disorders. However, the relation of the presence of this extra crease to any particular disease as yet is unknown.

The extra crease differs from white lines described by Cherrill (1950) inasmuch as the former separates epidermal ridges and disrupts local ridge configuration, with consequent localized deficiency of ridge formation.

The little fingers with extra creases showed no radiological evidence of anomalies of the bones or joints, nor did they display any appreciable dyskinesia.

Family studies of the member of the present series indicate that this trait has no prominent hereditary penetrance or expressibility.

Acknowledgment The authors wish to extend heartfelt gratitude to Dr. M. Okajima, Professor of Legal Medicine, Tokyo Medical and Dental University, for valuable guidance to the initiation of this investigation and they are grateful to Prof. G. T. Bryan, University of Wisconsin Medical School, for reviewing the manuscript.

\section{REFERENCES}

Alter, M. 1966. Dermatoglyphic analysis as a diagnostic tool. Medicine 46: 35-56.

Cherrill, F. R. 1950. Finger prints and disease. Nature 166: 581-584.

DeJong, R. and Plato, R. V. 1967. Sickle cell hemoglobinopathy. An anatomic sign. Amer. J. Dis. Child. 113: 271-272.

Furuya, Y., Inoue, T., Katafuchi, K., Mizoguchi, Y. and Murakata, S. 1967. A case of transverse skin creases in the middle phalange of the right ring finger. Acta Crim. Japon. 33: 49-50.

Okajima, M. 1966. A dermatoglyphical study of metacarpophalangeal creases. Amer. J. Phys. Anthrop. 24: 371-380.

Preus, M. and Fraser, F. C. 1972. Dermatoglyphics and syndromes. Amer. J. Dis. Child. 124; 933-943. 